\title{
Chaos: Exact, mixing and weakly mixing maps
}

\author{
Mohammed Nokhas Murad Kaki \\ Math Department, School of Science, Faculty of Science and Science Education, University of Sulaimani, Sulaymaniyah,Iraq
}

Email address:

muradkakaee@yahoo.com

\section{To cite this article:}

Mohammed Nokhas Murad Kaki. Chaos: Exact, Mixing and Weakly Mixing Maps. Pure and Applied Mathematics Journal. Vol. 4, No. 2, 2015, pp. 39-42. doi: 10.11648/j.pamj.20150402.11

\begin{abstract}
In this work, I studied a new class of topological $\lambda$-type chaos maps, $\lambda$-exact chaos and weakly $\lambda$-mixing chaos. Relationships with some other type of chaotic maps are given. I will list some relevant properties of $\lambda$-type chaotic map. The existence of chaotic behavior in deterministic systems has attracted researchers for many years. In engineering applications such as biological engineering, and chaos control, chaoticity of a topological system is an important subject for investigation. The definitions of $\lambda$-type chaos, $\lambda$-type exact chaos, $\lambda$-type mixing chaos, and weak $\lambda$-type mixing chaos are extended to topological spaces. This paper proves that these chaotic properties are all preserved under $\lambda \mathrm{r}$-conjugation. We have the following relationships: $\lambda$-type exact chaos $\Rightarrow \lambda$-type mixing chaos $\Rightarrow$ weak $\lambda$-type mixing chaos $\Rightarrow \lambda$-type chaos.
\end{abstract}

Keyword: Chaos, $\lambda$-Type Exact, Mixing, Weakly $\lambda$-Type Mixing, Conjugacy

\section{Introduction}

In this paper, I introduced and studied new types of chaotic maps. This is intended as a survey article on chaos of a discrete system given by a $\lambda$-irresolute self-map of a compact topological space. On one hand it introduces postgraduate students to the study of new types of chaotic maps and gives an overview of results on the topic, but, on the other hand, it covers some of the recent developments. I introduced and defined a new type of chaotic map called $\lambda$-type chaotic and investigate some of its properties. Relationships with some other type of chaotic maps are given. I list some relevant properties of the $\lambda$-type chaotic map. Further, I introduced the notions of $\lambda$ - mixing mapping. I have proved that every $\lambda$ type chaotic map is a chaotic map but the converse not necessarily true, and that every $\lambda$-mixing map is a mixing map, but the converse not necessarily true. Further, I studied a new class of topological $\lambda$-type chaos maps, $\lambda$-exact chaos and weakly $\lambda$-mixing chaos. Relationships with some other type of chaotic maps are given. I will list some relevant properties of $\lambda$-type chaotic map. The existence of chaotic behavior in deterministic systems has attracted researchers for many years. In engineering applications such as biological engineering, and chaos control, chaoticity of a topological system is an important subject for investigation. The definitions of $\lambda$-type chaos, $\lambda$-type exact chaos, $\lambda$-type mixing chaos, and weak $\lambda$-type mixing chaos are extended to topological spaces. This paper proves that these chaotic properties are all preserved under $\lambda$ r-conjugation. We have the following relationships: $\lambda$-type exact chaos $\Rightarrow \lambda$-type mixing chaos $\Rightarrow$ weak $\lambda$-type mixing chaos $\Rightarrow \lambda$-type chaos.

\section{Preliminaries and Definitions}

To study the dynamics of a self-map $f: X \rightarrow X$ means to study the qualitative behavior of the sequences $\left\{f^{n}(x)\right\}$ as $n$ goes to infinity when $\mathrm{x}$ varies in $\mathrm{X}$, where $f^{n}$ denotes the composition of $f$ with itself $\mathrm{n}$ times:

Definition2.1.[7] By a topological system I mean a pair $(X, f)$, where $X$ is a locally compact Hausdorff topological space (the phase space), and $f: X \rightarrow X$ is a continuous function. The dynamics of the system is given by $x_{n+1}=f\left(x_{n}\right), x_{0} \in X, n \in \mathbf{N}$ and the solution passing through $x$ is the sequence $\left\{f\left(x_{n}\right)\right\}$ where $n \in \mathbf{N}$.

Definition 2.2 Let $\mathrm{x} \in \mathrm{X}$, then the set $\left\{x, f(x), f^{2}(x), \ldots\right\}$ is called an orbit of $\mathrm{x}$ under $f$ and is denoted by $O_{f}(x)$, so $O_{f}(x)$ is the set of points which occur on the orbit of $x$ at some positive time, and the sequence $x, f(x), f^{2}(x), \ldots$ is called the trajectory of $\mathrm{x}$.

Any point with dense orbit is called a transitive point. A point which isnot transitive is called intransitive.

Topological dynamics is concerned with the behavior of iterations of a continuous map $f$ from a space $X$ into itself. 
Suppose for some $x \in X$, sequence $x, f(x), f^{2}(x), \ldots$ converges to some point say $x_{0} \in X$, then we must have $f\left(x_{0}\right)=x_{0}$, because $f$ is continuous. Such points we call as fixed points. We say that the point $x$ is attracted by the fixed point $x_{0}$. The set of all points in $X$ attracted by $x_{0}$ is called the stable set or the basin of attraction of the fixed point $x_{0}$ and is denoted by $W_{f}\left(x_{0}\right)$. A fixedpoint $x_{0}$ is said to be attracting if its stable set is a neighborhood of it.

A point $x \in X$ is said to beperiodic if there exists a positive integer $n \in \mathbf{N}$ such that $f^{n}(x)=x$. The set of all periodic points of the map $f$ is denoted by $\operatorname{per}(f)$.

Recall that a subset $S$ is a $\Lambda$-set (resp. a V-set) if and only if it is an Intersection (resp. a union) of open (resp. closed) sets and that a subset $A$ of a topological space $\mathrm{X}$ is called a $\lambda$ open set (or $\lambda$-closed) if $A=L \cap F$, where $L$ is a $\Lambda$-set and $F$ is closed. Complements of $\lambda$-closed sets will be called $\lambda$-open.

Definition 2.3.[4]A function $f: X \rightarrow X$ is called $\lambda$ irresolute if the inverse image of each $\lambda$-open set is a $\lambda$-open set in X.

Example 2.4[7]Let $(\mathrm{X}, \tau)$ be a topological space such that $\mathrm{X}=\{\mathrm{a}, \mathrm{b}, \mathrm{c}$,$\} and \tau=\{\phi,\{\mathrm{a}\},\{\mathrm{a}, \mathrm{b}\}, \mathrm{X}\}$. We have $\lambda \mathrm{O}(\mathrm{X}, \tau)=\{\phi$, $\mathrm{X},\{\mathrm{a}\},\{\mathrm{c}\},\{\mathrm{a}, \mathrm{b}\},\{\mathrm{a}, \mathrm{c}\},\{\mathrm{b}, \mathrm{c}\}$.If I define the map $\mathrm{f}: \mathrm{X} \rightarrow \mathrm{X}$ as follows $\mathrm{f}(\mathrm{c})=\mathrm{a}, \mathrm{f}(\mathrm{b})=\mathrm{b}, \mathrm{f}(\mathrm{a})=\mathrm{c}$. Then $\mathrm{f}$ is $\lambda$-irresolute.

Definition 2.5. A topological space $(X, \tau)$ is irreducible if every pair of nonempty open subsets of the space $X$ has a nonempty intersection.

In the study of dynamics on a topological space, it is natural and convenient to break the topological space into its irreducible parts and investigate the dynamics on each part. The topological property that precludes such decomposition is called topological transitivity.

In [7], I introduced the definitions of topological $\lambda$-type transitive and topologically $\lambda$-mixing maps as follows:

Definition 2.6.(1)Let $(\mathrm{X}, \tau)$ be a topological space, $f: X \rightarrow X$ be $\lambda$-irresolute map, then the map $f$ is called $\lambda$ type transitive if for every pair of non-empty $\lambda$-open sets $U$ and $\mathrm{V}$ in $\mathrm{X}$ there is a positive integer $\mathrm{n}$ such that $f^{n}(U) \cap V \neq \varphi$.

(2) A point $x \in X$ is $\lambda$-recurrent if, for every $\lambda$-open set $\mathrm{U}$ containing $\mathrm{x}$, infinitely many $n \in \mathbf{N}$ satisfy $f^{n}(x) \in U$. Thus, recurrence means that, under the iteration of $f$, the point $\mathrm{x}$ returns to each of its $\lambda$-neighborhoods infinitely often.

(3) Two points $x, y \in X$ are $\lambda$-proximal if, for every $\lambda$ neighborhood $\mathrm{F}$ of the diagonal $X \times X$, infinitely many $n \in \mathbf{N}$ satisfy $\left(f^{n}(x), f^{n}(y) \in F\right.$.

Note that every $\lambda$-proximal is proximal but the convers is not true.

Definition 2.7.(1)Let (X, $\tau)$ be a topological space, $f: X \rightarrow X$ be $\lambda$-irresolute map, then the map $f$ is called topologically $\lambda$-mixing if, given any nonempty $\lambda$-open subsets $U, V \subseteq X \exists N \geq 1$ such that $f^{n}(U) \cap V \neq \phi$ for all $\mathrm{n}>\mathrm{N}$. Clearly if $f$ is topologically $\lambda$-mixing then it is also $\lambda$ transitive but not conversely.
(2) The map $f$ is $\lambda$-exact if, for every nonempty $\lambda$ - open set $U \subset X$, there exists some $n \in \mathbf{N}$ such that $f^{n}(U)=X$.

(3) The map $f$ is (topological) $\lambda$-type transitive (resp., $\lambda$ mixing) if for any two nonempty $\lambda$ - open sets $U, V \subset X$, there exists some $n \in \mathbf{N}$ such that $f^{n}(U) \cap V \neq \phi$. (resp., $f^{m}(U) \cap V \neq \phi$. for all $\left.m \geq n\right)$.

(4) The map $f$ is weak $\lambda$-mixing if $f \times f$ is $\lambda$-type transitive on $X \times X$.

(5) A $\lambda$-mixing map $f: X \rightarrow X$ is pure $\lambda$-mixing if and only if there exists $\lambda$ - open set $U \subset X$ such that $f^{n}(U) \neq X$ for all $n \geq 0$.

(6)The map $f: X \rightarrow X$ is $\lambda$-type chaotic if $f$ is $\lambda$-transitive on $X$ and the set of periodic points of $f$ is $\lambda$-dense in $X$.

(7) The map $f$ is called $\lambda$-type exact chaos (resp., $\lambda$-type mixing chaos and $\lambda$-type weakly mixing chaos) if $f$ is $\lambda$-exact (resp., $\lambda$-mixing and $\lambda$-weakly mixing) and $\lambda$-type chaotic map on the space $X$.

Definition 2.8[7] Two topological systems $(X, f)$ and $(Y, g)$ are said to be h-conjugate if there is a homeomorphism $h: X \rightarrow Y$ such that $h \circ f=g \circ h$.

Suppose we have two topological systems, $f: X \rightarrow X$, $g: Y \rightarrow Y$. When the two systems are topologically conjugate, then the dynamics of one system completely describe the dynamics of the other.

First of all, any property of topological systems must face the obvious question: Is it preserved under topological conjugation? That is to say, if $\mathrm{f}$ has property $\mathrm{P}$ and if we have a commutative diagram

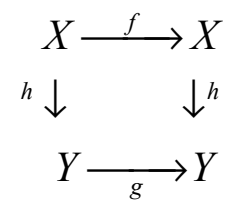

where $(X, f)$ and $(Y, g)$ are topological systems and $\mathrm{h}$ is a homeomorphism, then, is $\mathrm{g}$ necessarily has property $\mathrm{P}$ ? Certainly transitivity and the existence of dense periodic points are preserved as they are purely topological conditions.

\section{3. $\lambda$-Type Transitive Maps and Topological $\lambda r$-Conjugacy}

I introduced and defined $\lambda$-type transitive maps[7] and $\lambda-$ type minimal maps[7]. I will study some of their properties and prove some results associated with these new definitions. I investigate some properties and characterizations of such maps.

Definition 3.1 Recall that a subset A of a space $\mathrm{X}$ is called $\lambda$-dense in $\mathrm{X}$ if $C l_{\lambda}(A)=X$, we can define equivalent definition that a subset $\mathrm{A}$ is said to be $\lambda$-dense if for any $\mathrm{x}$ in $\mathrm{X}$ either $\mathrm{x}$ in $\mathrm{A}$ or it is a $\lambda$-limit point for $\mathrm{A}$.

Remark 3.2any $\lambda$-dense subset in $\mathrm{X}$ intersects any $\lambda$-open set in X.

Definition 3.3 A subset A of a topological space $(\mathrm{X}, \tau)$ is 
said to be nowhere $\lambda$-dense, if its $\lambda$-closure has an empty $\lambda$ interior, that is, $\operatorname{int}_{\lambda}\left(C l_{\lambda}(A)\right)=\phi$.

Definition 3.4 if for $x \in X$ the $\operatorname{set}\left\{f^{n}(x): n \in \mathbf{N}\right\}$ is dense in Xthenx is said to have a dense orbit. If there exists such an $x \in X$, then $f$ is saidto have a dense orbit.

Definition3.5. A function $f: X \rightarrow X$ is called $\lambda \mathrm{r}$ homeomorphism if $f$ is $\lambda$-irresolute bijective and $f^{-1}: X \rightarrow X$ is $\lambda$-irresolute.

Definition3.6Two topological systems $f: X \rightarrow X$, $x_{n+1}=f\left(x_{n}\right)$ and $g: Y \rightarrow Y, y_{n+1}=g\left(y_{n}\right)$ are said to be topologically $\lambda r$-conjugate if there is $\lambda r$-homeomorphism $h: X \rightarrow Y$ such that $h \circ f=g \circ h$ (i.e. $h(f(x))=g(h(x)))$.

We will call h a topological $\lambda r$-conjugacy.

Then I have proved some of the following statements:

1. $f$ and $g$ have the same kind of dynamics.

2. $h^{-1}: Y \rightarrow X$ is a topological $\lambda$ r-conjugacy.

3 . The map $f$ is $\lambda$-exact if and only if $g$ is $\lambda$-exact

4. The map $f$ is $\lambda$-mixing if and only if $g$ is $\lambda$-mixing

5. The map $f$ is $\lambda$-type chaotic if and only if $g$ is $\lambda$-type chaotic

6. The map $f$ is weakly $\lambda$-mixing if and only if $g$ is $\lambda$ weakly mixing.

Remark 3.7

If $\left\{\mathrm{x}_{0,}, \mathrm{x}_{1}, \mathrm{x}_{2}, \ldots\right\}$ denotes an orbit of $x_{n+1}=f\left(x_{n}\right)$ then $\left\{\mathrm{y}_{0}=\mathrm{h}\left(\mathrm{x}_{0}\right), \mathrm{y}_{1}=\mathrm{h}\left(\mathrm{x}_{1}\right), \mathrm{y}_{2}=\mathrm{h}\left(\mathrm{x}_{2}\right), ..\right\}$ yields an. In particular, $\mathrm{h}$ maps periodic orbits of $f$ onto periodic orbits of $g$.orbit of $\mathrm{g}$ since $y_{n+1}=h\left(x_{n+1}\right)=h\left(f\left(x_{n}\right)\right)=g\left(h\left(x_{n}\right)=g\left(y_{n}\right)\right.$, i.e. $f$ and $g$ have the same kind of dynamics.

I introduced and defined the new type of transitive[7] in such a way that it is preserved under topologically $\lambda \mathrm{r}$ conjugation.

In [7], we can see the proof of the following theorem and propositions.

Theorem 3.8.[7]For a $\lambda$-irresolute map $\mathrm{f}: \mathrm{X} \rightarrow \mathrm{X}$, where $\mathrm{X}$ is a topologicalspace, the following are equivalent:

1. $\mathrm{f}$ is topologically $\lambda$-type transitive;

2. Any Proper $\lambda$-closed subset $A \subset X \ni f(A) \subseteq A$ is nowhere $\lambda$-dense;

3. $\forall \mathrm{A} \subseteq \mathrm{X} \ni \mathrm{f}(\mathrm{A}) \subseteq \mathrm{A}, \mathrm{A}$ is either $\lambda$-dense or nowhere $\lambda$-dense;

4. Any subset $A \subseteq X \ni f^{-1}(A) \subseteq A$ with non-empty $\lambda$ interior is $\lambda$-dense.

Proposition3.9[7] if $\mathrm{f}: \mathrm{X} \rightarrow \mathrm{X}$ and $g: Y \rightarrow Y$ are $\lambda r$ conjugated by the $\lambda$ r-homeomorphism $h: Y \rightarrow X$. Then for all $\mathrm{y} \in \mathrm{Y}$ the orbit $O_{g}(y)$ is $\lambda$-dense in $\mathrm{Y}$ if and only if the orbit $O_{f}(h(y)) o f h(y)$ is $\lambda$-dense in $\mathrm{X}$.

Proposition3.10[7] Let $\mathrm{X}$ be a $\lambda$-compact space without isolated point, if there exists a $\lambda$-dense orbit, that is there exists $\mathrm{x}_{0} \in X$ such that the set $O_{f}\left(x_{0}\right)$ is $\lambda$-dense then the function $\mathrm{f}$ is $\lambda$-type transitive .

\section{Chaos in Product Topological Spaces}

Let $(X, f)$ be a topological system. A map $f: X \rightarrow X$ is called $\lambda$-type chaotic, if it is topological $\lambda$-type transitiveand, its periodic points are $\lambda$-dense in $X$ [8], i.e. every non-empty $\lambda$-open subset of $\mathrm{X}$ contains a periodic point. (A point $x \in X$ is called periodic if there exists $n \geq 1$ with $f^{n}(x)=x$ ) The set of all periodic points of $f$ denoted by $\operatorname{Per}(f)$.

Now, given two maps $f: X \rightarrow X$ and $g: Y \rightarrow Y$ on topological spaces $\mathrm{X}$ and $\mathrm{Y}$. respectively, consider their product $f \times \mathrm{g}: X \times \mathrm{Y} \rightarrow X \times \mathrm{Y}, \quad(\mathrm{f} \times \mathrm{g})(\mathrm{x}, \mathrm{y})=(\mathrm{f}(\mathrm{x}), \mathrm{g}(\mathrm{y}))$, with product topology on $\mathrm{X} \times \mathrm{Y}$

Lemma4.1Let $(X, f),(Y, g)$ be topological systems. The set of periodic points of $f \times \mathrm{g}$ is $\lambda$-dense in $X \times Y$ if and only if, for both of $f$ and $g$, the sets of periodic points in $\mathrm{X}$ and $\mathrm{Y}$ are $\lambda$-dense in $\mathrm{X}$, respectively Y.

Proof: Assume that the set of periodic points of $f$ is $\lambda$ dense in $\mathrm{X}$ (i.e. $\left.C l_{\lambda}(\operatorname{Per}(f))=X\right)$ and the set of periodic points of $\mathrm{g}$ is $\lambda$-dense in $\mathrm{Y}$ (i.e. $\left.\mathrm{Cl}_{\lambda}(\operatorname{Per}(g))=Y\right)$. We have to prove that the set of periodic points of $f \times \mathrm{g}$ is $\lambda$-dense in $X \times \mathrm{Y}$. Let $W \subset X \times \mathrm{Y}$ be any non-empty $\lambda$-open set. Then there exist non-empty $\lambda$-open sets $U \subset X$ and $V \subset Y$ with $U \times V \subset W$. By assumption, there exists a point $x \in U$ such that $f^{n}(x)=x$ with $n \geq 1$. Similarly, there exists $y \in V$ such that $g^{m}(y)=y$ with $m \geq 1$. For $\mathrm{p}=(\mathrm{x}, \mathrm{y}) \in \mathrm{W}$ and $\mathrm{k}=\mathrm{mn}$ we get

$$
\begin{aligned}
(f \times g)^{k}(p) & =(f \times g)^{k}(x, y) \\
& =\left(\left(f^{k}(x), g^{k}(y)\right)=(x, y)=p\right.
\end{aligned}
$$

Therefore $\mathrm{W}$ contains a periodic point and thus the set of periodic points of $f \times \mathrm{g}$ is $\lambda$-dense in $X \times \mathrm{Y}$.

Conversely let $\mathrm{U} \subset \mathrm{X}$ and $\mathrm{V} \subset \mathrm{Y}$ be non-empty $\lambda$-open subsets. Then $U \times \mathrm{V}$ is a non-empty $\lambda$-open subset of $X \times \mathrm{Y}$. As the set of the periodic points of $f \times g$ is $\lambda$-dense in $X \times \mathrm{Y}$, there exists a point $\mathrm{p}=(\mathrm{x}, \mathrm{y}) \in \mathrm{U} \times \mathrm{V}$ such that $(\mathrm{f} \times \mathrm{g})^{\mathrm{n}}(\mathrm{x}, \mathrm{y})=\left(\left(\mathrm{f}^{\mathrm{n}}(\mathrm{x}), \mathrm{g}^{\mathrm{n}}(\mathrm{y})\right)=(\mathrm{x}, \mathrm{y})\right.$ for some $\mathrm{n}$. From the last equality we obtain $f^{n}(x)=x$ for $\mathrm{x} \in \mathrm{U}$ and $g^{n}(y)=y$ for $y \in V$.

$\lambda$-denseness of periodic points carries over from factors to products. But, topological $\lambda$-type transitivity may not carry over to products. The converse of this situation is however true:

Lemma 4.2 Let $\mathrm{f}: \mathrm{X} \rightarrow \mathrm{X}$ and $\mathrm{g}: \mathrm{Y} \rightarrow \mathrm{Y}$ be maps and assume that the product $f \times \mathrm{g}$ is topological $\lambda$-type transitive on $\mathrm{X} \times \mathrm{Y}$. Then the maps $\mathrm{f}$ and $\mathrm{g}$ are both topological $\lambda$ type transitive on $\mathrm{X}$ and $\mathrm{Y}$ respectively.

Proof: We have to prove the $\lambda$-transitivity of $f$; the $\lambda$ transitivity of $g$ can be proved similarly. Let $U_{1}, V_{1}$ be non- 
empty $\lambda$-open sets in $X$. Then the sets $U=U_{1} \times Y$ and $V=V_{1} \times Y$ are $\lambda$-open in $X \times \mathrm{Y}$. As $f \times \mathrm{g}$ is $\lambda$ type transitive, there exists a positive integer $\mathrm{n}$ such that $(f \times g)^{n}(U) \cap V \neq \phi$. From the equalities:

$$
\begin{aligned}
(f \times g)^{n}(U) \cap V & =\left[f^{n}\left(U_{1}\right) \times g^{n}(Y)\right] \cap\left[V_{1} \times Y\right] \\
& =\left[f^{n}\left(U_{1}\right) \cap V_{1}\right] \times\left[g^{n}(Y) \cap Y\right] \neq \varphi,
\end{aligned}
$$

so $f^{n}\left(U_{1}\right) \cap V_{1} \neq \phi$. Thus $f$ is topological $\lambda$-type transitive.

Definition 4.3 Let $\mathrm{f}: \mathrm{X} \rightarrow \mathrm{X}$ be a map on the topological space $\mathrm{X}$. If for every nonempty $\lambda$-open subsets $U, V \subset X$ there exists a positive integer $n_{0}$ such that for every $n \geq n_{0}, f^{n}(U,) \cap V \neq \phi$ then $f$ is called topologically $\lambda$ type mixing.

It is clear that topological $\lambda$-type mixing implies topological $\lambda$-type transitive.There is an even stronger notion that implies topological $\lambda$-type mixing.

Definition 4.4Let $f: X \rightarrow X$ be a map on the space X. If for every nonempty $\lambda$-open subset $U \subset X$ there exists $n_{0} \in \mathbf{N} \backslash\{0\}$ such that for every $n \geq n_{0}, f^{n}(U)=X$, then $\mathrm{f}$ is called locally $\lambda$-type eventually onto.

Lemma 4.5 The product of two topologically $\lambda$-type mixing maps is topologically $\lambda$-type mixing.

Proof: Let $(X, f),(Y, g)$ be topological systems and $f, g$ be topologically $\lambda$-type mixing maps. Given $W_{1}, W_{2} \subset X \times Y$, there exists $\lambda$-open sets $U_{1}, U_{2} \subset X$ and $V_{1}, V_{2} \subset Y$, such that $U_{1} \times V_{1} \subset W_{1}$ and $U_{2} \times V_{2} \subset W_{2}$. By assumption there exist $n_{1}$ and $n_{2}$ such that $f^{k}\left(U_{1}\right) \cap U_{2} \neq \phi$ for $n \geq n_{1}$ and . $g^{k}\left(V_{1}\right) \cap V_{2} \neq \phi$ for $n \geq n_{2}$. For $\mathrm{n} \geq \mathrm{n}_{0}=\max \left\{\mathrm{n}_{1}, \mathrm{n}_{2}\right\}$ we get

$$
\begin{array}{r}
{\left[(f \times g)^{k}\left(U_{1} \times V_{1}\right)\right] \cap\left(U_{2} \times V_{2}\right)=\left[f^{k}\left(U_{1}\right) x g^{k}\left(V_{1}\right)\right] \cap\left(U_{2} x V_{2}\right)} \\
=\left[f^{k}\left(U_{1}\right) \cap U_{2}\right] x\left[g^{k}\left(V_{1}\right) \cap V_{2}\right] \neq \varphi
\end{array}
$$

Which means that $f \times g$ is topologically $\lambda$-type mixing.

We give some sufficient conditions for a product map to be $\lambda$-type chaotic.

Theorem 4.6: Let $\mathrm{f}: \mathrm{X} \rightarrow \mathrm{X}$ and $\mathrm{g}: \mathrm{Y} \rightarrow \mathrm{Y}$ be $\lambda$-type chaotic and topologically $\lambda$-type mixing maps on topological spaces $\mathrm{X}$ and $\mathrm{Y}$. Then $\mathrm{f} \times \mathrm{g}: \mathrm{X} \times \mathrm{Y} \rightarrow \mathrm{X} \times \mathrm{Y}$ is $\lambda$-type chaotic.

Proof: The map $\mathrm{f} \times \mathrm{g}$ has $\lambda$-dense periodic points by Lemma 4.1 and it is topologically $\lambda$-type mixing by Lemma 4.5 and hence topologically $\lambda$-type transitive. Thus the two conditions of $\lambda$-type chaos are satisfied.

\section{Conclusion}

There are the following results:

Proposition5.1

Topologically $\lambda$-type exact chaos $\Rightarrow \lambda$-type mixing chaos $\Rightarrow$ weak $\lambda$-type mixing chaos $\Rightarrow \lambda$-type chaos

\section{Proposition5.2}

If $f: X \rightarrow X$ and $g: Y \rightarrow Y$ are topologically $\lambda r$ conjugate. Then

(1) The map $f$ is $\lambda$-exact if and only if $g$ is $\lambda$-exact

(2) $f$ is weakly $\lambda$-mixing if and only if $g$ is weakly $\lambda$ mixing.

(3) $\mathrm{f}$ is $\lambda$-type chaotic on $\mathrm{X}$ if and only if $g$ is $\lambda$-type chaotic in $\mathrm{Y}$.

Lemma 5.3Let $(X, f),(Y, g)$ be topological systems. The set of periodic points of $f \times \mathrm{g}$ is $\lambda$-dense in $X \times Y$ if and only if, for both of $f$ and $g$, the sets of periodic points in $X$ and $\mathrm{Y}$ are $\lambda$-dense in $\mathrm{X}$, respectively $\mathrm{Y}$.

Lemma 5.4Let $f: X \rightarrow X$ and $g: Y \rightarrow Y$ be maps and assume that the product $f \times \mathrm{g}$ is topological $\lambda$-type transitive on $X \times \mathrm{Y}$. Then the maps $f$ and $\mathrm{g}$ are both topological $\lambda$ type transitive on $\mathrm{X}$ and $\mathrm{Y}$ respectively.

Lemma 5.5The product of two topologically $\lambda$-type mixing maps istopologically $\lambda$-type mixing.

Theorem 5.6Let $f: X \rightarrow X$ and $g: Y \rightarrow Y$ be $\lambda$-type chaotic and topologically $\lambda$-type mixing maps on topological spaces $\mathrm{X}$ and $\mathrm{Y}$. Then $\mathrm{f} \times \mathrm{g}: \mathrm{X} \times \mathrm{Y} \rightarrow \mathrm{X} \times \mathrm{Y}$ is $\lambda$-type chaotic.

\section{References}

[1] H. Maki, Generalized $\Lambda$-sets and the associated closure operator, The Special Issue in Commemoration of Prof. Kazusada IKED’S Retirement, 1 Oct. (1986), 139-146.

[2] F.G. Arenas, J. Dontchev and M. Ganster, On $\lambda$-sets and the dual of generalized continuity, Questions Answers Gen. Topology, 15 (1997), 3-13.

[3] M. Caldas and S. Jafari, On some low separation axioms via $\lambda$--open and $\lambda$-closure operator, Rend. Circ. Mat. di Palermo, 54(2005), 195-208.

[4] M. Caldas, S. Jafari and G. Navalagi, More on $\lambda$--closed sets in topological spaces, RevistaColombiana, 41 (2007), 355-369.

[5] S. G. Crossley and S. K. Hildebrand, Semi topological properties, Fund. Math. 74(1972) 233-254

[6] A. A. El-Atik, A study of some types of mappings on topological spaces, Master's Thesis, Faculty of Science, Tanta University, Tanta, Egypt 1997.

[7] Mohammed Nokhas Murad, New Types of $\Lambda$-Transitive Maps, International Journal ofResearch in Electrical and Electronics Engineering (ISTP-JREEE) ISSN: 2321-2667 Volume 2, Issue 5, Sept. 2013 pp. 19-24

[8] Mohammed Nokhas Murad, New types of chaotic maps on topological spaces, International Journal of Electrical and Electronic Science, American association for science and technology, (AASCIT) 2014; 1(1): 1-5, USA. 